\title{
Untersuchungen zur Spezifitätsfrage der heterosynaptischen Facilitation bei Aplysia californica*
}

\author{
B. JaHan-Parvar und R. v. BaumgarTeN \\ Neurophysiologische Abteilung des Mental Health Research Institutes \\ der Universität von Michigan, Ann Arbor/Mich., USA
}

Eingegangen am 17. März 1967

Summary. 1. Heterosynaptic facilitation (H.S.F.) could be obtained in $10-15 \%$ of all tested nerve cells in the abdominal and left pleural ganglion of Aplysia californica.

2. In all of the cells showing H.S.F. this phenomenon appeared not only after paired stimulation, but also when the test stimulus was independent of the priming stimulus. Cells which showed H.S.F. only after paired stimulation (specific H.S.F.) could not be found.

3. In most cases paired stimulation was more effective than priming alone, causing a larger H.S.F.

4. The degree of the H.S.F. depended largely on the right combination of test and priming nerve. Changing the test nerve and keeping the priming nerve constant could change the effect from inhibition to "no effect" or to H.S.F. The same could be observed by keeping the test nerve constant but changing the priming nerve. Homosynaptic facilitation appeared in all tested nerves.

5. In about $10 \%$ of the cells with H.S.F., the facilitation was preceded by an early partial depression of the test response. With sustained priming stimulation this depression gave way to a facilitation. After the end of the priming series a second peak of facilitation was reached, suggesting a release phenomenon.

6. When H.S.F. was repeated after a pause of $20 \mathrm{~min}$ the effect of the priming stimulus on the test response was potentiated.

7. It is concluded that in the recorded cells H.S.F. exibited unspecific as well as specific features. The unspecific sensitization could this represent useful background for possible discriminative functions of the specific H.S.F.

8. Some similarities between H.S.F. and conditioned responses are discussed.

Eine hinreichend starke Reizung eines beliebigen Nerven eines Ganglions von Aplysia führt in der Regel dazu, daß die synaptische Wirksamkeit (gemessen an der Größe des postsynaptischen excitatorischen Potentials nach schwacher Testreizung) mehrerer oder aller anderen Nerven des Ganglions für mehrere Minuten zunimmt. Diese von KaNDeI u. TAUC [7,8] als heterosynaptische Facilitation (H.S.F.) beschriebene Erscheinung scheint bei oberflächlicher Betrachtung so unspezifisch zu sein, daß sie zur Erklärung spezifischer Lernvorgänge

\footnotetext{
* Mit Unterstützung der Deutschen Forschungsgemeinschaft.
} 
wenig nützt. Es handelt sich vielmehr um eine Art Sensitivierung, bei der das postsynaptische Substrat nach dem „Priming“-Reiz stärker auf jeglichen Testreiz reagiert als zuvor. Immerhin haben KandeL u. TAUC [7] in ganz seltenen Ausnahmefällen auch drei Neurone gefunden, bei denen eine Prüfung ergab, daß heterosynaptische Facilitation nur nach gepaarter Reizung eintrat. Vergleichbar mit den klassischen bedingten Reflexen mußte hier der „bedingte" Testreiz dem ,unbedingten “ Primingreiz zeitlich einige hundert Millisekunden vorausgehen [5]. Da uns das spezifische Verhalten dieser seltenen Neurone für die Neurophysiologie der Lernvorgänge besonders interessant erschien, haben wir im folgenden versucht, die spezifischen Aspekte der heterosynaptischen Facilitation näher von den unspezifischen Eigenschaften abzugrenzen.

Trotz eingehender Suche konnten wir bis jetzt aber kein vollständig spezifisches Neuron in Aplysia finden. Dafür wurden aber auch bei den scheinbar unspezifischen Neuronen gewisse spezifische Eigenschaften festgestellt. Aufgrund dieser Beobachtungen erscheint uns die Annahme berechtigt, daß die Spezifität bei der H.S.F. eine graduelle Eigenschaft darstellt, welche dem unspezifischen Sensitivierungsprozeß überlagert ist.

\section{Methodik}

Bezüglich Einzelheiten sei auf die vorausgehende Arbeit [2] verwiesen. Kurz zusammengefaßt handelt es sich darum, daß eine Nervenzelle des Abdominal- oder des linken Pleuralganglions in Aplysia californica in vitro intracellulär abgeleitet wird, während ein konstanter Testreiz auf einen, afferente Fasern enthaltenden, ,Testnerven" in gleichmäßigen zeitlichen Abständen gegeben wird. Dieser Testreiz wurde entweder mit einem nachfolgenden Primingreiz zeitlich mit 350 msec $A b$ stand starr gekoppelt (gepaarte Reizung), oder der Primingreiz wurde zum Vergleich unter Beibehaltung desselben abgeleiteten Neurons in zeitlich unabhängigen Abstand gegeben, wobei das Intervall zum letzten und zum nächsten Testreiz mindestens 4 sec betrug (ungepaarte Reizung). Ein Schaltkasten erlaubte einen schnellen Wechsel des Priming- und des Testreizes auf je einen beliebigen von den fünf, im Falle des isolierten Z.N.S., 20 Nerven, die auf ebenso viele bipolare Reizelektroden gelegt worden waren. Bei gleichbleibenden Primingnerven konnte so der Testnerv gewechselt oder bei gleichbleibendem Testnerv gegen den Primingnerven in einigen Fällen gegenseitig ausgewechselt werden. Theoretisch ergeben sich so 25 verschiedene Prüfungsmöglichkeiten pro Neuron. Praktisch konnte aber nur ein Teil dieser Kombination geprüft werden, da die lange Versuchsdauer nicht vereinbar mit vergleichbaren Test- und Primingantworten war und außerdem mit langfristigen Potenzierungen (siehe unten) gerechnet werden mußte.

\section{Ergebnisse \\ 1. Unspezifische H.S.F.}

Es wurden über 86 möglichst verschiedene Neurone im Abdominalganglion, und über 20 mal die Riesenzelle des linken Pleuralganglions intracellulär abgeleitet. Diese Neurone wurden daraufhin geprüft, ob H.S.F. an ihnen überhaupt ausgelöst werden konnte, und wenn ja, 
ob und inwieweit diese nur nach gepaarter Reizung, oder auch nach ungepaarter Reizung eintrat.

Nicht alle Nervenzellen im Abdominalganglion zeigten eine, mit der verwendeten Methode nachweisbare Form der H.S.F. In Übereinstimmung mit den Befunden von KaNdeL u. TaUC [7] schätzten wir die
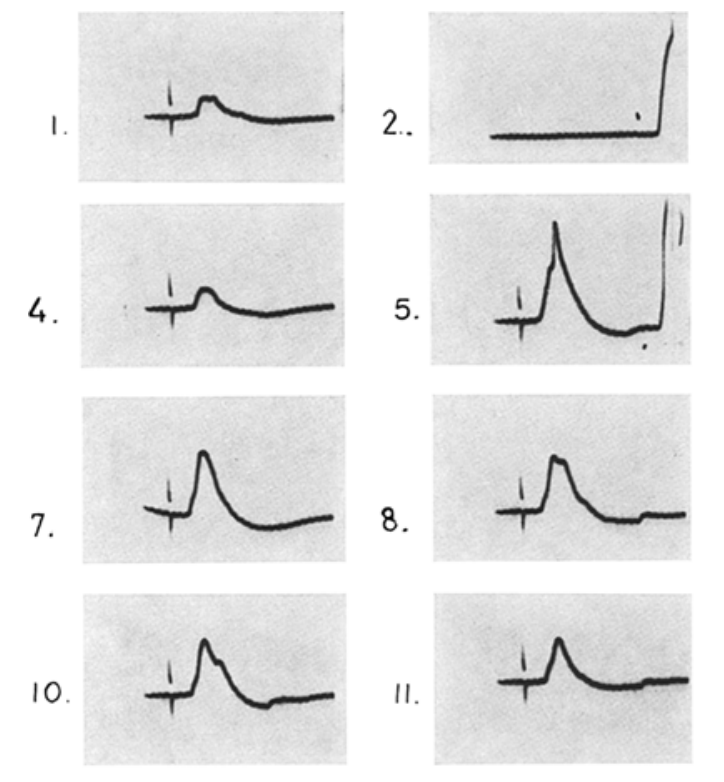

3.

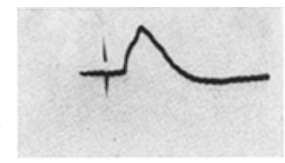

6.
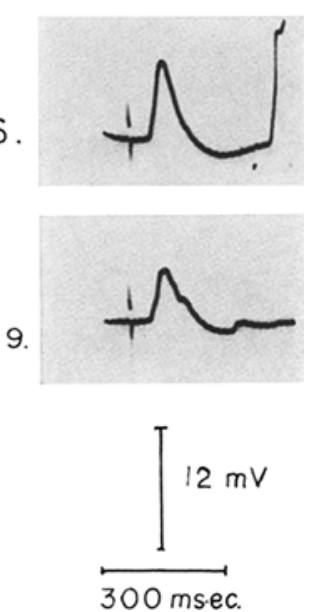

Abb.1. Heterosynaptische Facilitation an einer Zelle des Abdominalganglions. Nichtidentifizierte Zelle links dorsal der Riesenzelle des Abdominalganglions. Testreiz: linkes Connectiv, Primingreiz: Siphonnerv. 1 Kontrolle; 2 Priming alleine; $310 \mathrm{sec}$ später schwache H.S.F.; 4 Kontrolle nach Rückgang des Test-E.P.S.P.s auf den Ausgangswert; 510 sec nach der ersten gepaarten Reizung starke H.S.F.; 6 H.S.F. 40 sec später nach der vierten gepaarten Reizung; 7 bis II. Weitere Testantworten in Abständen von 10 sec. Überdauern der H.S.F. nach abgestellter Primingreizung und allmählicher Rückgang

Neurone, welche H.S.F. zeigten, auf nur 10-20\% der Gesamtpopulation, wobei zu berücksichtigen ist, daß unsere Ableitung eine Selektion der größten Zellen darstellt, und daß außerdem vielleicht weit entfernt liegende Synapsen bei der Ableitung des Zellsomas nicht erfaßt werden können. Die Riesenzelle des rechten oberen Quadranten zeigte regelmäßig eine H.S.F., ebenso die ihr homologe Riesenzelle des linken Pleuralganglions.

Alle geprüften Zellen, an denen sich überhaupt H.S.F. auslösen ließ, zeigten diese auch, wenn der Primingreiz zeitlich unabhängig vom Testreiz erfolgte (ungepaarte Reizung). Der zeitliche Abstand zwischen den 
Reizen wurde in diesen Fällen unregelmäBig verabfolgt und schwankte zwischen 4 und 10 sec. Der verwendete Primingreiz war überschwellig und löste seinerseits ein orthodrom übertragenes Aktionspotential aus. Besondere Aufmerksamkeit wurde dem Areal links der Riesenzelle des rechten oberen Quadranten geschenkt, in welchem KANDEL u. TAUC [7] drei unidentifizierte Zellen gefunden hatten, die spezifisch reagierten.

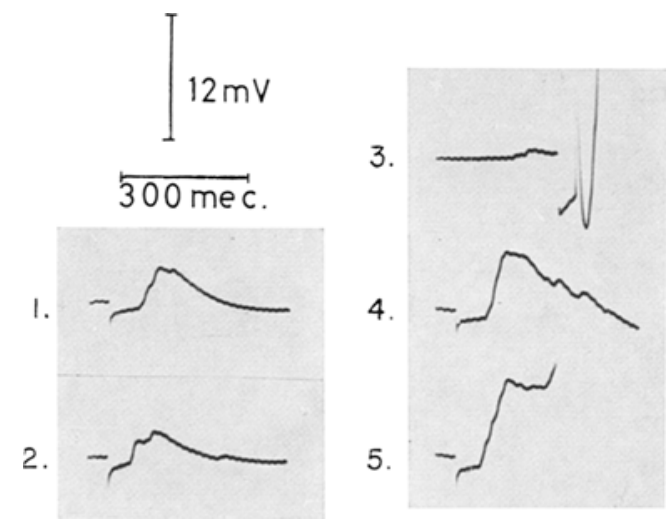

Abb.2. Spezifitästest an der Riesenzelle des linken Pleuralganglions. Primingnerv: linkes Connectiv. 1 Kontrolle der Testantwort im linken Pedalnerven; 2 Kontrolle der Testantwort im rechten Connectiv; 3 Primingreizung alleine des linken Connectives; 4 H.S.F. im linken Pedalnerven 10 sec nach Priming; 5 H.S.F. im rechten Connectiv 20 sec nach Priming. Beide Testnerven waren unspezifisch von einem Primingnerven aus gefördert

Wir waren aber auch hier nicht in der Lage, irgendeine Zelle zu finden, bei welcher der Primingstimulus, wenn alleine und stark genug angewandt, nicht auch schon unspezifische Förderereffekte erzeugte. Abb. 1 zeigt als Beispiel eine der unidentifizierten Zellen dieses Areals, welche eine heterosynaptische Facilitation sowohl nach ungepaarter, als auch nach gepaarter Reizung zeigte. Die Reihenfolge, in der hier gepaarte und ungepaarte Reizung angewandt wurde, erwies sich als unwesentlich, wenn nur ein oder wenige Primingreize verwendet wurden. In Abb. 2 ist eine Spezifitätsprüfung an der Riesenzelle des linken Pleuralganglions durchgeführt worden. Die Zelle reagiert hier ebenfalls unspezifisch, da Priming alleine die Testantwort in mehr als einem Nerven förderte.

2. Stärkerer Bahnungseffekt bei gepaarter als bei ungepaarter Reizung

Diese Befunde führten zu der nächsten Frage, nämlich, ob bei den scheinbar unspezifisch reagierenden Zellen nicht wenigstens ein quantitativer Unterschied zwischen den Effekten gepaarter und ungepaarte 
Reizung besteht. Die experimentelle Prüfung dieser Annahme ergab tatsächlich, daß sich in den meisten Zellen durch gepaarte Reizung eine H.S.F. mit höherer Amplitude erzeugen ließ als bei getrennter Reizung. Dieses läßt sich gut auf Abb.1 erkennen, wenn man die Amplitude der Testantwort nach Priming alleine (Abb.1, Teil 3) mit derjenigen nach gepaarter Reizung (Abb.1, Teil 5) vergleicht. Das gleiche galt auch für
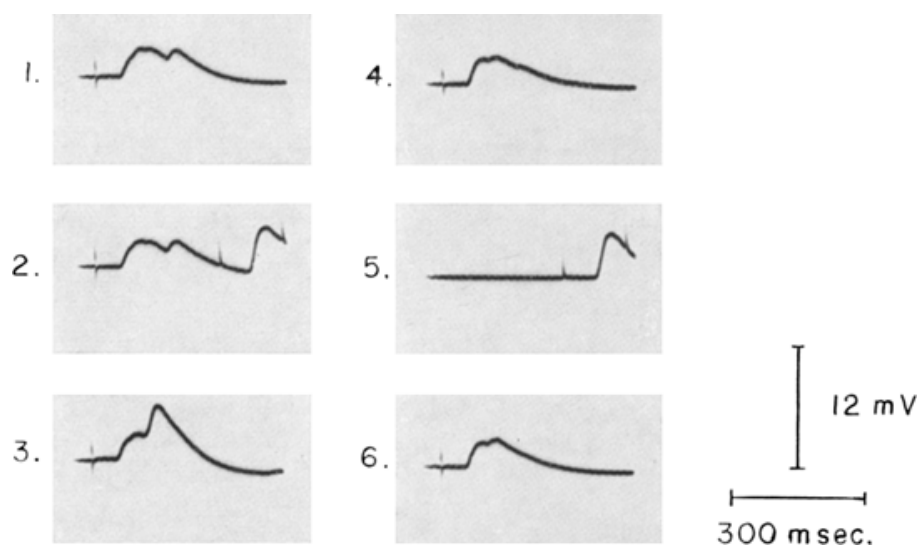

Abb.3. Vergleich des Effeltes gepaarter und ungepaarter Reizung an der R.O.Q. Riesenzelle. Testnerv: linkes Connectiv, Primingnerv: Perikardialast des Genitalnerven. Der Primingreiz war gerade überschwellig für ein Aktionspotential eingestellt. 1 Kontrolle der Testantwort; 2 gepaarte Reizung; 3 H.S.F. 10 sec nach gepaarter Reizung; 4 Kontrolle der Testantwort nach Abklingen der H.S.F.; 5 Priming alleine (ungepaarte Reizung); 6 keine H.S.F. nach Priming alleine. Die Ableitungen zeigen, da $\beta$ gepaarte Reizung wirksamer als ungepaarte Reizung ist, und daß auch die R.O.Q. Riesenzelle bei schwachem Primingreiz partiell spezifisch reagieren kann

die Riesenzelle des rechten oberen Quadranten, deren unspezifische Reaktionsform schon bekannt war. Wurde hier der Primingreiz gerade so schwach eingestellt, daß die unspezifische Reaktion nach Priming alleine ausblieb, so blieb die H.S.F. nur nach gepaarter Reizung übrig (Abb.3). Bei alleiniger Kenntnis dieses Befundes wäre man versucht, das Neuron in die Reihe der spezifischen Zellen einzureihen. In manchen Fällen genügte ein einziger Rechteckreiz, was zeigt, daß Tetanisierung keine notwendige Voraussetzung der H.S.F. ist, sondern daß, wenn auch mit geringerem Effekt, ein einzelner Reizschlag ausreichend sein kann, um H.S.F. hervorzurufen. Die Beobachtung, daß gepaarte Reizung effektiver ist als ungepaarte Reizung wurde in sechs Fällen auf Film registriert und in mindestens acht weiteren Fällen auf dem Oscillographen beobachtet. Sie war für den einzelnen Fall meistens wiederholbar und unabhängig 
von der Reihenfolge. In schätzungsweise $20-30 \%$ der abgeleiteten Zellen ließ sich der Effekt nicht erzielen. Da die anfängliche Einstellung der Voltstärke des Test- und Primingreizes für diesen Versuch kritisch ist und außerdem nur Präparationen mit konstantem Test- und PrimingReizerfolg geeignet sind, enthält dieser letztere Prozentsatz auch rein technische Versager.

\section{Abhängigkeit der H.S.F. von der Kombination verschiedener Nerven}

Obwohl ein Primingreiz, wenn er nur stark genug ist, Förderung in den E.P.S.P.s einer Mehrzahl von Testnerven hervorruft, so bestehen hier doch deutliche graduelle Unterschiede. Manche Testnerven ließen sich durch Priming anderer Nerven überhaupt nicht fördern, unabhängig davon, ob gepaarte oder nicht gepaarte Reizung angewandt wurde (Abb.4). Nach Austausch des Primingnerven mit dem Testnerven an der selben abgeleiteten Zelle trat H.S.F. ein. Eine besonders ausgiebige Förderung resultierte bei beiden Nerven, wenn der Primingreiz sowohl als auch der Testreiz auf denselben Nerven gelegt wurden (homosynaptische Facilitation). Der Versuch zeigte, daß auch ein Nerv, der keine H.S.F. bot, einer homosynaptischen Förderung seines E.P.S.P.s durchaus fähig war. Der Zeitverlauf dieser Förderung war in den wenigen Fällen, in denen wir homosynaptisch reizten, nicht wesentlich abgekürzt, doch reichen unsere diesbezüglichen Befunde bei der Variabilität der H.S.F. zu einem statistischen Vergleich mit der heterosynaptischen Facilitation [7] nicht aus.

Abb.4. Austausch von Priming- und Testnerv. Zelle: Nicht identifizierte Zelle links der R.O.Q. Riesenzelle. Die Reizstärke für den Testnerven war jeweils deutlich überschwellig für ein E.P.S.P., die für den Primingnerven, oder für die homosynaptische Reizung gerade überschwellig für ein Aktionspotential eingestellt. Der Strahlablauf ist nur in dieser Abbildung bei laufendem Film schräg nach unten gerichtet. A Testnerv: Branchialnerv, Primingnerv: Siphonnerv, keine H.S.F.; B Testnerv: Siphonnerv, Primingnerv: Branchialnerv, H.S.F.; C Testnerv: Branchialnerv, Primingreizung desselben Nerven, gute homosynaptische Facilitation; D. Testnerv: Siphonnerv, Primingreizung desselben Nerven, gute homosynaptische Facilitation. Die Ableitungen zeigen, daß eine unwirksame Nervenkombination durch den Austausch von Test- und Primingreiz wirksam werden kann

Abb.5. Wechsel des Testnerven bei konstantem Primingnerven. Primingnerv in allen Ableitungen: Siphonnerv. Der Testreiz wurde gewechselt und jeweils so stark bemessen, daß ein Kontroll-E.P.S.P. gleicher Größenordnung resultierte. Die oberste Ableitung entspricht jeweils der Kontrolle, die mittlere der ersten und einzigen gepaarten Reizung, die unterste ist 10 sec nach der gepaarten Reizung aufgenom-

men worden. A Testnerv: linkes Connectiv, keine H.S.F.; B Testnerv: rechtes Connectiv, leichte H.S.F.; C Testnerv: Genitalnerv, Frühhemmung, die später (nicht gezeigt) in eine leichte H.S.F. überging; D. Testnerv: Branchialnerv, gute H.S.F. 


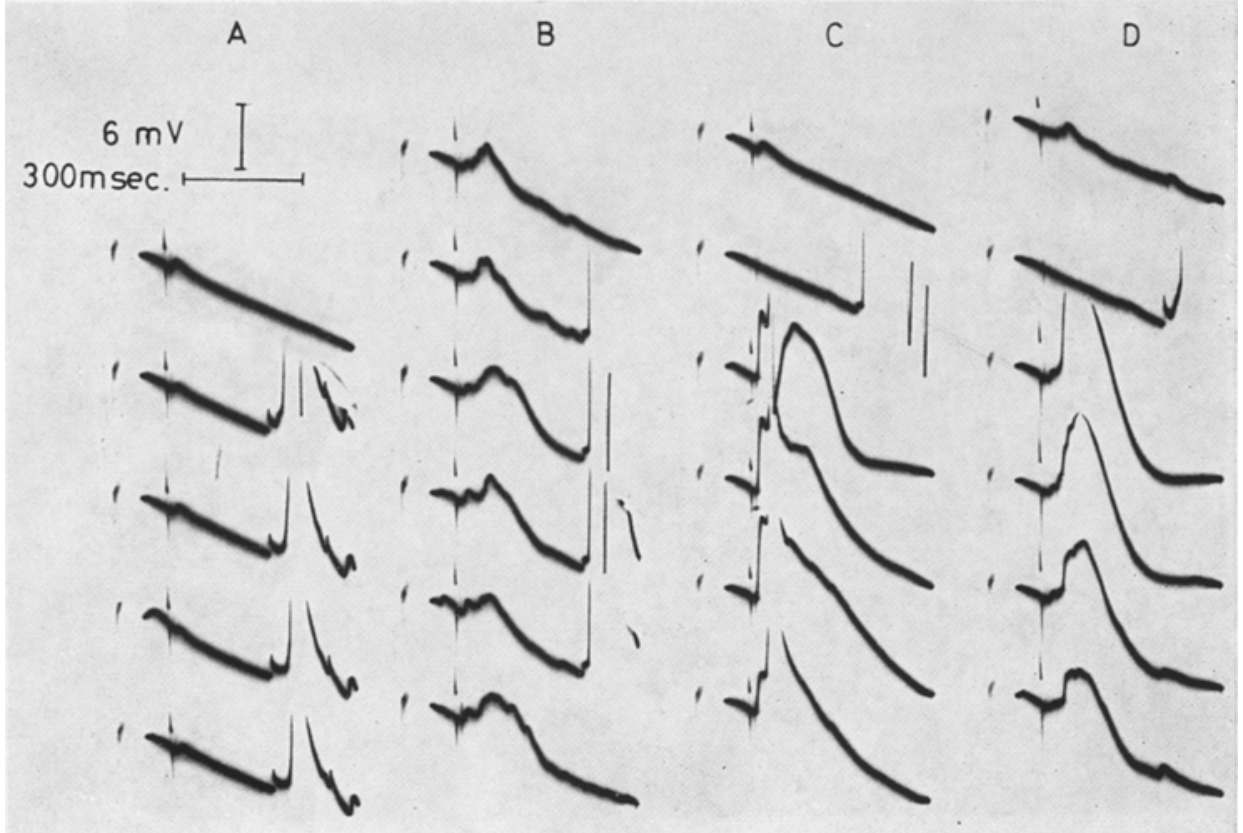

Abb.4 (Legende siehe S.352)

A

B

C

D
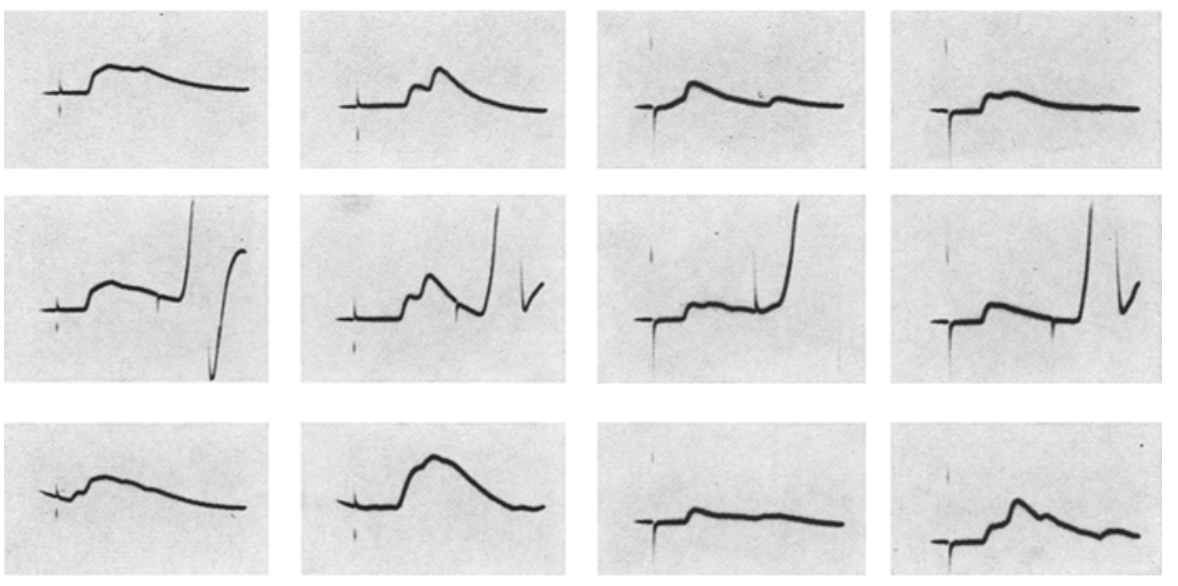

Abb.5 (Legende siehe S. 352)

$$
\frac{I 6 \mathrm{mv}}{300 \mathrm{msec} .}
$$


1.

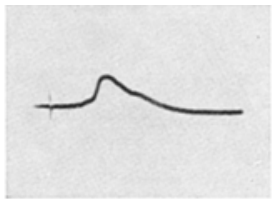

4.

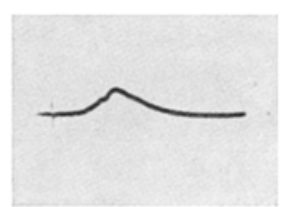

2.

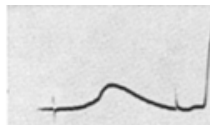

5.

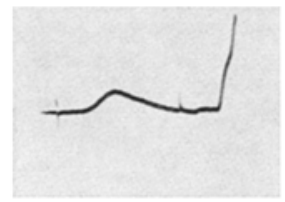

3.

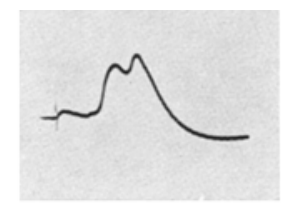

6.

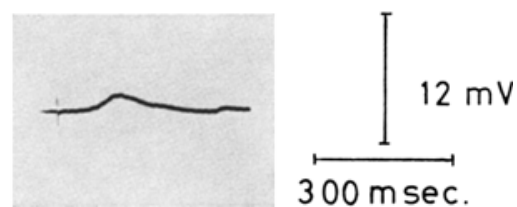

Abb.6. Wechsel des Primingnerven bei konstantem Testnerven. Testnerv in allen Ableitungen: Branchialnerv. 1 Kontrolle; 2 gepaarte Reizung, Primingnerv: Genitalast; 380 sec nach gepaarter Reizung, deutliche H.S.F.; 4 Kontrolle $10 \mathrm{~min}$ danach; 5 gepaarte Reizung, Primingnerv: Perikardialast; 680 sec nach gepaarter Reizung, kein Effekt (auch früher oder später kein Effekt). Die Abbildung zeigt, daß bei konstantem Testnerven der Wechsel des Primingnerven eine Veränderung der H.S.F. bewirken kann

Wurde unter Beibehaltung des Primingnerven nur der Testnerv gewechselt, so änderte sich auch die Größe der H.S.F. An derselben abgeleiteten Zelle zeigten manche Testneurone Förderung, andere gar keinen Effekt, andere sogar Hemmung (Abb.5). Ebenso konnte sich die H.S.F. wesentlich ändern, wenn unter Beibehaltung des Testnerven nur der Primingnerv gewechselt wurde (Abb.6).

Die Befunde zeigen, daß die Kombination des, ,richtigen“ Primingnerven mit dem , richtigen" Testnerven bedeutungsvoll für das Ausmaß der H.S.F. ist. Die Kombinationsabhängigkeit konnte ausnahmslos in mehr als 30 geprüften Fällen beobachtet werden.

\section{Frühhemmung und Zweigipfeligkeit der H.S.F.}

Bei einem Teil der abgeleiteten Zellen mit heterosynaptischer Facilitation fiel in etwa $10 \%$ der Fälle auf, daß unmittelbar nach Beginn des Primings eine vorübergehende Depression des Test-E.P.S.P.s unter den Kontrollwert eintrat, welche dann nach etwa 10 sec einer H.S.F. Platz machte (Abb.7). Diese Anfangshemmung konnte dann am besten dargestellt werden, wenn die Testreize einander in geringem Abstand von nur 5 sec folgten. Sie entzog sich wahrscheinlich der Beobachtung in den Fällen, in denen der erste Testreiz nach einer noch längeren Zeitdauer dem Priming folgte. Die wirkliche Dauer dieser Hemmung konnte deshalb nicht bestimmt werden, weil die sich entwickelnde H.S.F. eine vielleicht noch überdauernde Hemmung maskieren würde. Dennoch 
A

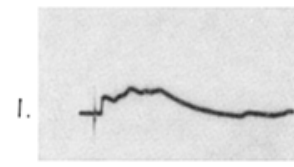

2.

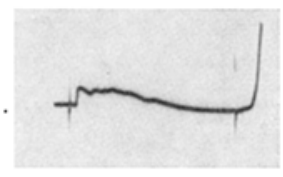

3.

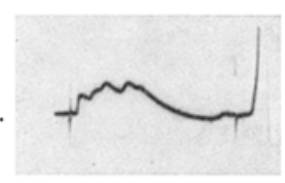

4.

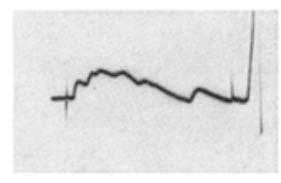

5.

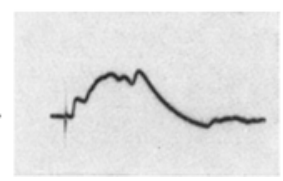

B

6.

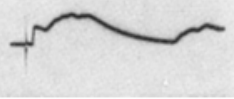

7.

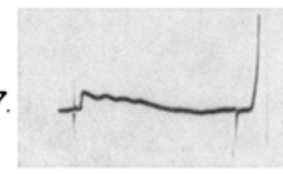

8.

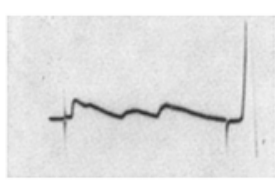

9

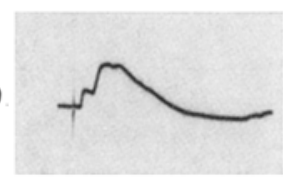

10

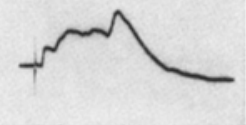

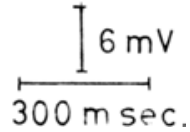

Abb.7. Frühhemmung der Testantwort bei der H.S.F. Zelle: R.O.Q. Riesenzelle, Testreiz: Branchialnerv, Primingreiz: Siphonnerv. A und B sind zwei gleichverlaufende Experimente an derselben Präparation. 1 und 6 Kontrollen; 2 and 7 5 sec nach der ersten gepaarten Reizung. Frühhemmung, das Test-E.P.S.P. ist unter den Ausgangswert gefallen; 3 bis 5 und 8 bis 10 die Frühhemmung geht in eine H.S.F. über

könnte ein weiterer Befund für das Fortbestehen der Hemmung über die ganze Primingperiode sprechen. Wenn gegen Ende der Primingperiode die H.S.F. bereits wieder im Absinken war, kam es in mehreren Präparationen unmittelbar nach dem Abschalten des Primings zu einem sofortigen Wiederanstieg der H.S.F., der zu einem zweiten Gipfel führte (Abb.8). Die unmittelbare Abhängigkeit des Zeitpunktes dieses zweiten Wiederanstiegs vom Ende der Primingserie in verschiedenen Präparationen zeigt, daß es sich nicht um einen Späteffekt, sondern eher um das plötzliche Ende eines primingabhängigen, konkurrierenden Hemmvorganges oder vielleicht auch um das Erholen von einer durch den fortgesetzten Reiz bedingten Erschöpfung handelt. 


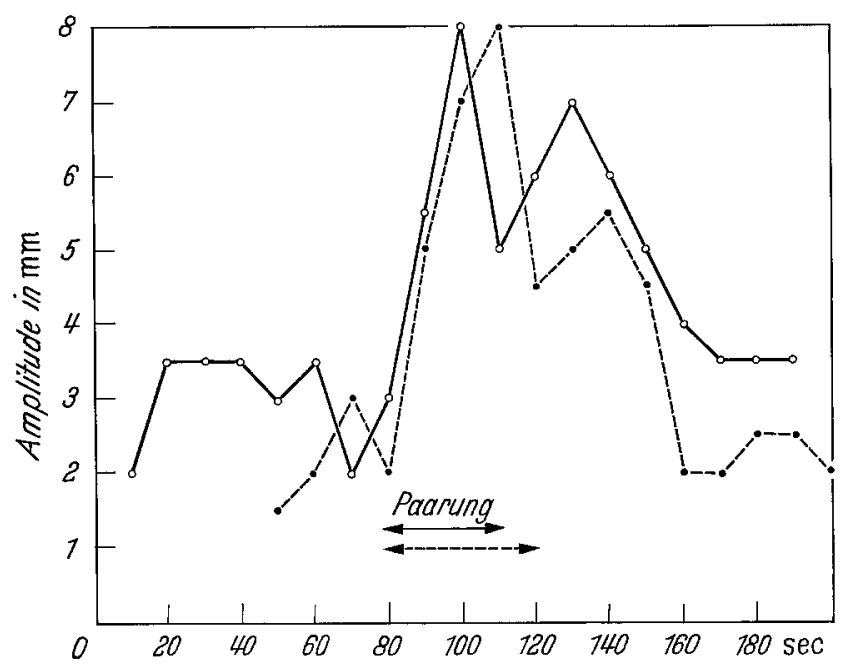

Abb.8. Zweigipfeligkeit der H.S.F. Die ausgezogene und die gestrichelte Kurve stammen von zwei verschiedenen Experimenten und sollen die Konstanz des Befundes bei verschiedenen Versuchstieren demonstrieren. Ausgezogene Kurve. Zelle: R.O.Q. Riesenzelle, Testnerv: Genitalnerv, Primingnerv: Branchialnerv. Gestrichelte Kurve. Zelle: nicht identifizierte Zelle links der R.O.Q. Riesenzelle, Testnerv: Branchialnerv, Primingnerv: Siphonnerv. Beide Kurven zeigen einen leichten Abfall der H.S.F. noch während der Primingperiode. Unmittelbar nach Abschalten der Primingreizung kommt es, nach Art eines „release-Phänomens“, zum Wiederanstieg der H.S.F., die dann, wie üblich, langsam wieder abklingt

\section{Langfristige Bahnungseffekte}

Bei Wiederholung der heterosynaptischen Facilitation in längeren zeitlichen Abständen trat eine Potenzierung des Bahnungseffektes ein. Die Kurve der Abb.9 stammt von einer unspezifischen H.S.F. an der R.O.Q. Riesenzelle des Abdominalganglions. Nach einer Reizpause von 15 min wurde die Primingreizung mit denselben Reizparametern wiederholt. Der Effekt übertraf deutlich den der ersten Reizung. Dieser Potenzierungseffekt wurde in weiteren zwei Fällen beobachtet, aber nicht registriert. In einem weiteren registrierten Falle nahm die H.S.F. progessiv ab. Eine zur Zeit in Gang befindliche Versuchsserie widmet sich speziell dieser Fragestellung.

\section{Besprechung der Ergebnisse}

Unsere Beobachtungen über die H.S.F. in dieser, wie auch in der vorangehenden Arbeit stehen in keinem Widerspruch zu den Befunden von KaNdel u. TAUC [7,8]. Die Pionierarbeit dieser Autoren kann durch die vorliegende Arbeit bestätigt und in einigen Punkten erweitert werden. 


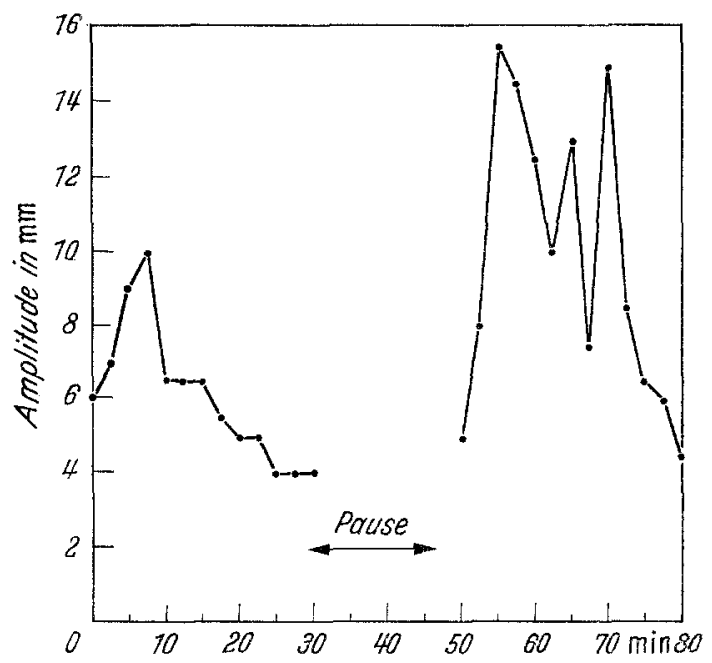

Abb.9. Potenzierte H.S.F. bei wiederholter Anwendung. Zelle: R.O.Q. Riesenzelle, Testnerv: Siphonnerv, Primingnerv: Branchialnerv. Die Kurvenpunkte entsprechen ungepaarter Testreizung. Fortgesetzte Reizung des Primingnerven alleine führte zunächst zu einer unspezifischen H.S.F., die nach etwa $10 \mathrm{~min}$ in zunehmende Erschöpfung überging. Wenn nach 15 min Pause dieselbe Reizung wiederholt wird, so ist der so erzeugte Anstieg der Testantwort sehr viel höher als beim erstenmal

Hinsichtlich der Spezifität der H.S.F. führte uns unsere Versuchsanordnung zu einer etwas verschiedenen Auffassung. Da wir nach aus. giebiger Prüfung keine sich ausschließlich spezifisch verhaltende Zelle gefunden haben, sehen wir vorläufig keinen Anlaß, an deren Existenz zu glauben. Bei der nur geringen Zahl (nur drei) der von den genannten Autoren geprüften und als spezifisch beschriebenen Zellen, halten wir es aufgrund unserer zusätzlichen Befunde über die unterschiedliche Wirksamkeit verschiedener Test- und Primingneurone für möglich, daß bei der Prüfung dieser drei Neurone zufälligerweise ungünstige Kombinationen zwischen Testnerv und Primingnerv vorlagen.

Die hier vorgelegten Ergebnisse lassen aber den Schluß zu, daß eine Art partieller Spezifität der H.S.F. bei den meisten oder allen Neuronen existiert. Diese Spezifität ist der gleichzeitig an denselben Neuronen bestehenden unspezifischen Reaktionsweise überlagert. Sie drückt sich darin aus, daß gepaarte Reizung wirksamer ist als ungepaarte Reizung, und daß an derselben Zelle verschiedene Kombinationen von Testnerv und Primingnerv jeweils verschiedene Wirksamkeit hinsichtlich der H.S.F. haben.

Die neuronale Grundlage dieser Beobachtungen wird aufgrund der in der vorigen Arbeit [2] beschriebenen Befunde verständlicher. Die Testantwort ist 
danach aus mehreren Einzel-E.P.S.P.s, die von verschiedenen Testneuronen stammen, zusammengesetzt. Einige davon haben - so wurde aufgrund der Erschöpfungskurven vermutet - eine direkt fördernde Verbindung von der Primingseite her, andere nicht. Eine direkte Verbindung würde bedeuten, daß ein solches Testneuron hinsichtlich des Termines seines Feuerns sowieso mit der Primingseite gekoppelt ist. Gepaarte Reizung von außen ist hier nicht nötig, und Priming alleine ruft ,,unspezifische " H.S.F. hervor. Die anderen Testeinheiten desselben abgeleiteten Neurons, welche keine fördernde Verbindung von der Primingseite her haben, bedürfen allerdings der künstlich gepaarten Reizung von außen, um H.S.F. zu zeigen.

Der Grad der Spezifität steht nach dieser Hypothese im umgekehrten Verhältnis zur Zahl der direkt fördernden Zwischenverbindungen. Er ist offensichtlich verschieden für verschiedene Neurone und innerhalb desselben Neurons unterschiedlich für verschiedene Nervenkombinationen. Diese Erklärungsmöglichkeit würde bedeuten, daß H.S.F. immer nur dann eintritt, wenn Test- und Primingneurone in zeitlicher Nachbarschaft entladen werden.

Die Beobachtung, daß an manchen Zellen eine frühe Hemmphase der H.S.F. vorausgeht und vielleicht sogar während der ganzen Primingperiode anhält, spricht dafür, daß fördernde und hemmende Vorgänge am gleichen Neuron bei Reizung derselben Afferenzen konkurrieren können. Einzelne inhibitorische postsynaptische Potentiale haben wir allerdings nicht beobachtet, doch würden diese auch durch die gleichzeitigen E.P.S.P.s überschattet werden. Auch an ein Konkurrieren von präsynaptischer Hemmung mit H.S.F. könnte gedacht werden, da nach den Befunden TAUC's [10] präsynaptische Hemmung in Aplysia keine Seltenheit darstellt.

Eine äußerlich ganz ähnliche Zweigipfeligkeit wurde von Hurbard [6] auch bei der posttetanischen Potenzierung der neuromuskulären Endplatte beobachtet und dort auf Transmitterverarmung während der Tetanisierung zurückgeführt. In unserem Falle ist eine frühe Verarmung zum Zeitpunkte der Anfangshemmung unwahrscheinlich, doch könnte sie die Ursache des Absinkens vor dem zweiten Gipfel sein.

Es besteht bis jetzt noch kein sicherer Anhalt dafür, daß die H.S.F. einen an Lernvorgängen und am Gedächtnis beteiligten Mechanismus darstellt. Andererseits aber besteht begründeter Verdacht, daß bestimmte Formen des Kurzgedächtnisses ein unentbehrliches Glied in der Kette zu anderen Mechanismen des Langzeitgedächtnisses darstellen (Agranoff [1], BRoAdbENT [3]). Unter den wenigen bekannten in Frage kommenden neurophysiologischen Korrelaten des Kurzgedächtnisses, nimmt der spezifische Anteil der H.S.F. gegenüber anderen Mechanismen eine besondere Rolle ein: Die Bahnung einer synaptischen Verbindung alleine durch häufige Benutzung, wie sie z. B. bei der posttetanischen Potenzierung der Fall ist, erklärt noch keinen bedingten Reflex (OLDs [9]). Bei der spezifischen heterosynaptischen Facilitation hingegen wird eine anlagemäßig vorhandene, aber unterschwellige synaptische Verbindung erst dadurch voll und in überdauernder Weise funktionsfähig gemacht, daß Impulse von einer anderen 
afferenten Seite her zur Hilfe kommen. Zum Unterschied von der einfachen konvergierenden synaptischen Summation handelt es sich bei der spezifischen H.S.F. um eine Bahnung, welche nur für bestimmte Reizkombinationen gilt und auch nach dem Abklingen der lokalen synaptischen Potentiale noch für mehrere Minuten anhält. Die Überlappung unspezifischer Sensitivierung mit spezifischen Förderungen an demselben Neuron erscheint sinnvoll, weil dabei die ankommenden spezifischen Erregungen bereits allgemein gesteigerte Hintergrunds-Aktivität antreffen und so leichter überschwellig werden. Unser Befund, daß potenzielle Unterschiede in der Ausbildung der H.S.F. bei verschiedenen Nervenkombinationen bestehen, läßt ebenfalls einen Vergleich mit den klassischen bedingten Reflexen zu. Hier wie dort baut sich der antrainierte Effekt auf dem Hintergrund des bereits vorhandenen angeborenen und erlernten Verhaltensrepertoires auf. Eine dritte formelle Ähnlichkeit der H.S.F. mit den bedingten Reflexen betrifft die Abhängigkeit beider von der Erregung zweier afferenter Reflexbögen. Wird der unbedingte Reiz beim bedingten Reflex im efferenten (motorischen) Teil verabfolgt, so tritt trotz äußerlicher Gleichartigkeit des unbedingten Reizerfolges kein bedingter Reflex ein (Tracy [11], Hilgard u. Allex [4]). In Analogie dazu tritt auch keine H.S.F. ein, wenn die abgeleitete postsynaptische Zelle direkt intracellulär gereizt wird [8] oder wenn sie durch einen ,,ungeeigneten“ Primingnerven zum Aktionspotential gebracht wird (Abb.6).

Es wäre Aufgabe nachfolgender Untersuchungen, zu prüfen, ob bei häufiger Wiederholung der H.S.F. über einen längeren Zeitraum chronische Veränderungen der Testantwort im Sinne einer Informationsspeicherung auftreten oder nicht. Ein erster Anfang mag vielleicht unsere Beobachtung sein, daß in mehrminutigen Abständen wiederholte H.S.F. zu potenzierter Wirkung führt. Die Frage ist auch hier, ob dynamisches Kurzgedächtnis auf neuronaler Ebene in strukturell fixiertes Langzeitgedächtnis übergehen kann. Die Tatsache, daß Aplysia-Präparationen mehrere Tage lang abgeleitet werden können [2], läßt solche Versuche über einen längeren Zeitraum durchführbar erscheinen.

\section{Literatur}

1. Agranoff, B. W., R. E. Davis, and J. J. Brink: Chemical studies on memory fixation in goldfish. Brain Res. 1, 303-309 (1966).

2. Baumgarten, R. v., u. B. Jahan-Parvar: Beitrag zum Problem der heterosynaptischen Facilitation in Aplysia californica. Pflügers Arch. ges. Physiol. 295, 328-346 (1967).

3. Broadbent, D. E.: Successive responses to simultaneous stimuli. Quart. J. exp. Psychol. 8, 145-152 (1956).

4. HLLARD, E. R., and M. K. Alr.EN: An attempt to condition finger reactions based on motor point stimulation. J. gen. Psychol. 18, 203-207 (1938). 
5. Hilgard, E.R., and D. G. MarquIs: Conditioning and learning, pp. 155-160. New York: Appleton-Croft 1961.

6. HUbBaRd, J. I.: Repetitive stimulation at the mamalien neuromuscular junction, and the mobilization of transmitter. J. Physiol. (Lond.) 169, 641-662 (1963).

7. KANDEL, E. R., and L. TAUC: Heterosynaptic facilitation in neurones of the abdominal ganglion of Aplysia depilans. J. Physiol. (Lond.) 181, 1-27 (1965).

8. - - Mechanism of heterosynaptic facilitation in the giant cell of the abdominal ganglion of Apylysia depilans. J. Physiol. (Lond.) 181, 28-47 (1965).

9. OldS, J.: Discussion of the paper of Dr. Ecolets. Brain Mechanismus and Learning. Springfield, Ill.: Ch. C. Thomas 1961.

10. Tadc, L.: Praesynaptic inhibition in the abdominal ganglion of Aplysia. J. Physiol. (Lond.) 181, 282-307 (1965).

11. Tracy, F. W.: Experiments on the establishment of conditioned motor responses. M. A. Thesis, Ohio State University, p. 322 (1927).

Professor Dr. R. v. BaumaarTen

Mental Health Research Institute University of Michigan

Ann Arbor/Michigan, U.S.A. 